Article

\title{
Bio-Inspired Adhesive Footpad for Legged Robot Climbing under Reduced Gravity: Multiple Toes Facilitate Stable Attachment
}

\author{
Zhongyuan Wang ${ }^{1,2}$ (D), Zhouyi Wang ${ }^{2}$ (D) , Zhendong Dai ${ }^{2, *}$ (i) and Stanislav N. Gorb ${ }^{3}$ \\ 1 The 28th Research Institute of China Electronics Technology Group Corporation, Nanjing 210007, China; \\ wangzy051@163.com \\ 2 Institute of Bio-inspired Structure and Surface Engineering, Nanjing University of Aeronautics and \\ Astronautics, Nanjing 210016, China; wzyxml@nuaa.edu.cn \\ 3 Department Functional Morphology and Biomechanics, Zoological Institute of the University of Kiel, \\ Kiel 24118, Germany; sgorb@zoologie.uni-kiel.de \\ * Correspondence: zddai@nuaa.edu.cn
}

Received: 13 November 2017; Accepted: 11 January 2018; Published: 15 January 2018

\begin{abstract}
This paper presents the design of a legged robot with gecko-mimicking mechanism and mushroom-shaped adhesive microstructure (MSAMS) that can climb surfaces under reduced gravity. The design principle, adhesion performance and roles of different toes of footpad are explored and discussed in this paper. The effect of the preload velocity, peeling velocity and thickness of backing layering on the reliability of the robot are investigated. Results show that pull-force is independent of preload velocity, while the peeling force is relying on peeling velocity, and the peel strength increased with the increasing thickness of the backing layer. The climbing experiments show that the robot can climb under mimic zero gravity by using multiple toes facilitating adhesion. The robot with new type of footpads also provides a good platform for testing different adhesive materials for the future space applications.
\end{abstract}

Keywords: legged robot; mushroom-shaped adhesive microstructure; adhesion performance; reduced gravity; climbing

\section{Introduction}

The extraordinary locomotory abilities of geckos, insects and spiders, which are attributed to striking adhesive setae segmented into scansors or pads on the undersides of the toes or legs, have been well-known for many centuries [1]. The outstanding climbing performances of animals inspired the engineers and researchers for the design of artificial systems, such as adhesive materials, which in turn enabled the construction of climbing robots that are capable of climbing smooth walls and ceiling [2-4].

Inspired by hairy footpads of various animals in nature, fiber arrays with different end shapes such as mushrooms, asymmetric spatulae and concave structures have been developed [5-11]. By using the fibrillar surfaces, several robotic prototypes with tank-like treads and rotary legs have been reported. Mini Whegs robot [12] was equipped with mushroom-shaped adhesive microstructure (MSAMS) made of polyvinyl siloxane (PVS), using four mulit-spoke wheel-leg appendages. This 100-120 g heavy robot easily climbs smooth vertical and even inverted surfaces. Waalbot [13], equipped with rotating compliant legs, is able to climb on smooth vertical walls using angled polyurethane fiber arrays.

Due to the limitation of the mechanism these robots can only work on relatively flat areas of the surface using relatively uniform motion pattern. However, legged climbing robots may increase their potential to adapt their gaits to uneven surfaces and allow for more instantaneous control of stability, if we can push or pull them effectively during the intermittent contact with the substrate at 
each step. Several groups have developed legged robots that use dry adhesives and capable of such a contact formation/breakage control [14-16]. One of the most impressive robots is the Stickybot [17], which utilizes directional polymer stalks (DPS) to effectively climb vertical surfaces, such as glass, plastic, and polished wood panels. Although the climbing abilities of the robot on ground and wall have been reported, its ability to perform locomotion under zero gravity, when contact formation by DPS is not supported by the gravity force, is still unknown.

Legged climbing robot involves the controlled application of forces during the leg-substrate contact and release, in order to propel the body forwards. The achievement of strong attachment and easy removal of the adhesive is the critical issues, when we apply the adhesive to the robot's footpad. Previous studies have considered the effect of the peel angle, the length and the shape of the peel region of the adhesive itself on the adhesion performance. However, the influences of the shape and stiffness of the footpad itself are rarely studied.

IBSS_Gecko is a legged mechanism for a gecko-inspired robot, which is first introduced by Dai and Sun (Figure 1a) [18]. Using a single toe which is composed of a spring-loaded runner sheet coated with pressure-sensitive adhesive at each foot, IBSS_Gecko_6 was able to climb on smooth vertical surface [19]. The improvements presented in this work are built upon the basic design principles of the robot. Inspired by the geckos, we introduce here a footpad with multiple toes (Figure 1b) and biomimetic MSAMS (Figure 1c-e) [10], to increase adaptability and stability of the climbing robot. The legged robot with only one toe is difficult to cope with the different environments. And the novel fibrillar adhesives may enable the robot to climb on surfaces regardless of the gravity.

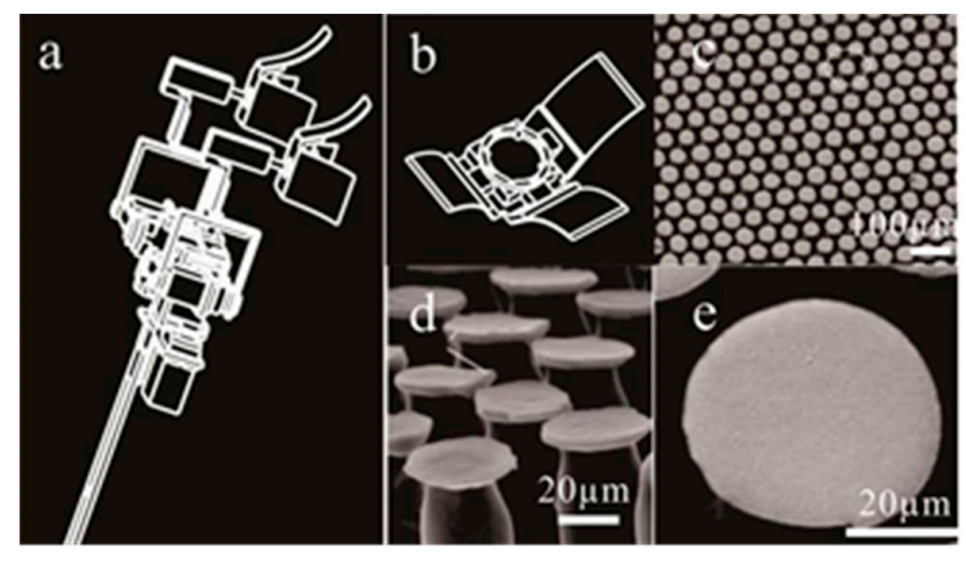

Figure 1. Structural hierarchy of the biologically inspired robot adhesive system. (a) Gecko-inspired robot; (b) Footpad of the robot; (c-e) Biomimetic mushroom-shaped adhesive microstructure (MSAMS) made of PVS (reproduced with permission from [10]. Copyright the Royal Society, 2007).

This paper is organized in the following manner. Section 2 briefly reviews the biomechanics of a gecko climbing on a surface. Based on the biological principle in micro and macro scales, we develop a gecko-inspired footpad. The manner of footpad with strong attachment and easy-removal properties is designed and tested in Section 3. Furthermore, experiments on the IBSS_Gecko on the challenged condition-reduced gravity is designed, analyzed and discussed in Section 4. Finally, conclusions and future work are reported in Section 5 .

\section{Footpad Design}

Biomechanics of a gecko climbing on a surface reveals the utilization of a particular Y-configuration in both the first and fifth toes and opposite feet (Figure 2a) [20-26]. The opposite feet are pulling inward toward the center of mass, generating large in-plane forces to ensure the angle of pulling force vector towards the substrate remains small. The geckos favor setal attachment at two different 
hierarchical characteristic sizes of toes and feet. Additionally, geckos with multiple toes can avoid detachment by aligning some of their toes opposite to the force vector [24,27].

IBSS_Gecko_6 robot consists of a rigid body and four legs (Figure 2b). The mass of the robot is about $500 \mathrm{~g}$, while the length and width is $240 \mathrm{~mm}$ and $100 \mathrm{~mm}$, respectively. Each leg has two linkages and three degrees of freedom. Three motors drive the leg to move it in the three dimensional space. The first motor (Hitec HS-85MG, Chungcheongbuk-do, South Korea) controls the rotational motion. The second and the third motors (Hitec HS-65MG, Chungcheongbuk-do, South Korea) control the motion and position of the foot. An integrated flexure in the foot (ball hinge and four springs) allows for up to 15 degrees of pitch, roll and yaw misalignment to obtain alignment and full contact with substrate. This design largely reduces required precision of footpad's trajectory and greatly increases the adaptability of the robot to different surfaces.

To mimic the function of the gecko's footpad, a biomimetic footpad was developed (Figure 2b). It has similar geometric appearance as the gecko's footpad, including three rectangular toes pointing to three directions. Every toe is composed of supporting layer, backing layer and adhesive layer. The adhesive layer is attached to one side of the supporting layer, while the backing layer is attached to the other side of the supporting layer. The supporting layer was superimposed with the backing layer, so that it can prevent the adhesive from deforming irreversibly. Obviously, the thickness of the backing layer is largely influence adhesion strength of the footpad. However, the role of the backing layer thickness on adhesion performance for legged climbing robot has not been investigated in detail so far.

a

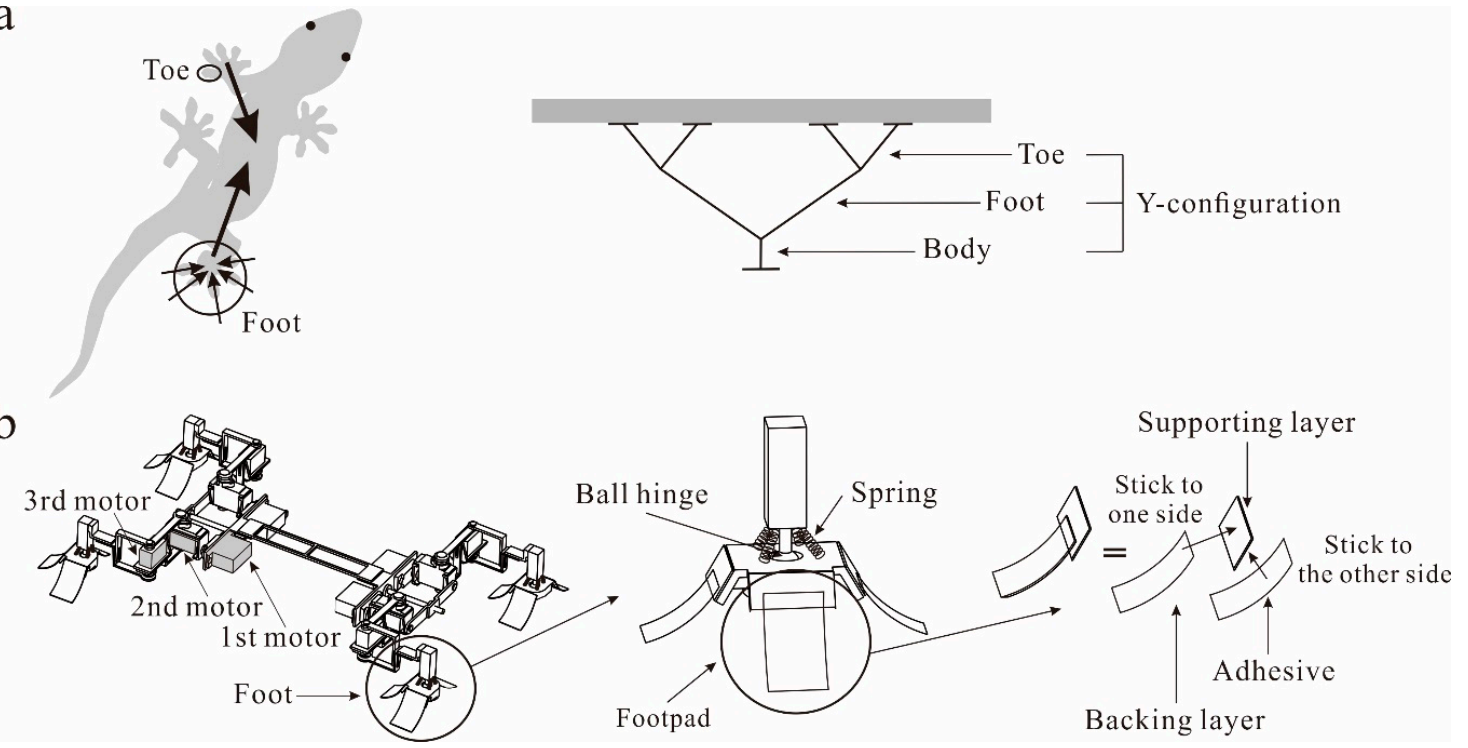

Figure 2. (a) Arrangement of toes in the gecko walking on a ceiling surface reveals the utilization of a particular Y-configuration in both toes and feet; (b) Mechanical structure of the legged robot and its footpad.

\section{Adhesion Performance}

The footpad movements consist of two phases: attachment and detachment. Attachment and detachment time determine the speed and influence stability of the robot. For example, for pressure-sensitive adhesives (PSA), if the footpad attaches just for a short time, it may only generate a small adhesion force. Furthermore, if the PSA-footpad tries to detach at once, it must overcome a strong adhesion force. Reducing the peeling force effectively at detachment is essential for keeping the stability of the robot. In this part of the locomotory cycle, both the pull-off test and peeling test will examine how attachment and detachment times influence adhesion performance. Furthermore, we study here the backing layer thickness effect on the adhesion performance of the pad. 


\subsection{Pull-Off Test}

A pull-off test was conducted using Nano and Micro Tribometer UMT-2 (Campbell, CA, USA). MSAMS specimen in the form of discs of $3 \mathrm{~mm}$ diameter was used as a dry adhesive. The specimen was attached to an aluminum block, fixed on the force transducer. The specimen and block were perpendicular to the load direction. The specimen was brought into contact and detached by linear motion of the translation stage, which was oriented perpendicularly to the glass surface (average roughness $R a=3.3 \pm 2.1 \mathrm{~nm}$ ) (Figure 3a). The pull-off force was measured, while the translation stage moved at different attach velocities $\left(V_{0}\right)$ with the same preload and the same detach velocity $\left(V_{t}=0.2 \mathrm{~mm} / \mathrm{s}\right)$. Contact time between the MSAMS specimen and the glass substrate was $5 \mathrm{~s}$.

The pull-off force measured between the PVS specimen and the glass substrate is presented in Figure $3 \mathrm{~b}$. The result reveals that the pull-off force on the glass substrate is not only independent of the load force [10], but also relies on the load velocity. The result indicates that for the robot, the footpad of this kind does not need much load control: the pad just needs to contact the surface, in order to produce substantial adhesion force and meet the requirement of the attaching phase. This characteristic of MSAMS is extremely beneficial for applying it at zero gravity, when the robot cannot rely on gravity to load the adhesive pad.
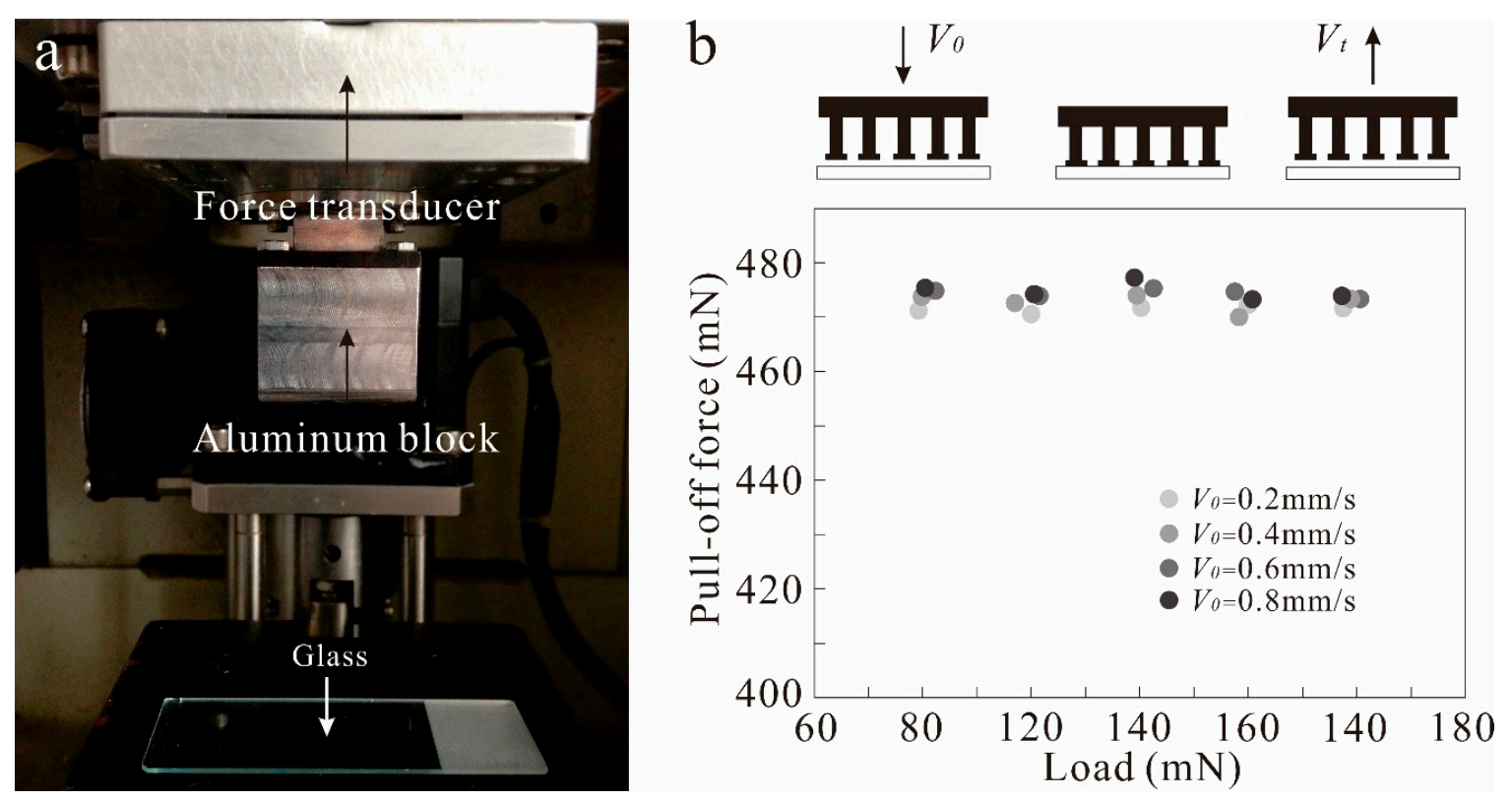

Figure 3. (a) Image of the setup used to measure the pull-off force; (b) The pull-off force measured between mushroom-shaped adhesive microstructure (MSAMS) and glass as a function of load at different load velocities.

\subsection{Peeling Test}

A peeling strength analysis is performed according to the Kendall's peeling model [28]:

$$
\left(\frac{F}{b}\right)^{2} \frac{1}{2 E d}+\left(\frac{F}{b}\right)(1-\cos \theta)-R=0
$$

where $F$ is the peeling force, $d$ is the thickness of the adhesive, $b$ is the width of the adhesive, $E$ is the Young's modulus of the adhesive, $\theta$ is the peeling angle, and $R$ is the energy required to fracture a unit area of interface.

Kendall's peeling model demonstrates that peel strength increases with increasing Young's modulus of the backing layer. When the PVS is peeled off from a substrate, fibrills are stretched and deformed until the tension stress surpasses the pull-off stress. Under this condition, detachment 
is started. For the fibrillar surface, the stretching velocity of the fibril influences the resulting peel force [29]. Previous studies have shown that the relationship between the peel force and the peel velocity at the same peel angle can be described by [30-32]

$$
\frac{F}{b}=k V^{n}
$$

where $k$ is a function of the peel angle and is related to the thickness of the adhesive, $V$ is peel velocity, and $n$ is a constant related to the intrinsic properties of the adhesive: this parameter is often called adhesive energy.

Our testing platform was set up to estimate the effect of peel velocity and the thickness of the adhesive on the peel strength (Figure 4). Pieces of the MSAMS dry adhesive $(20 \mathrm{~mm} \times 100 \mathrm{~mm})$ with different thickness of backing layer (Polyvinyl chloride plastic) were attached to a smooth glass surface (average roughness $R a=3.2 \pm 1.9 \mathrm{~nm}$ ) and preloaded with different weights. The peel angle was adjusted by fixing the glass surface at various tilted angles. Different peel velocities are obtained by changing the loading weights.
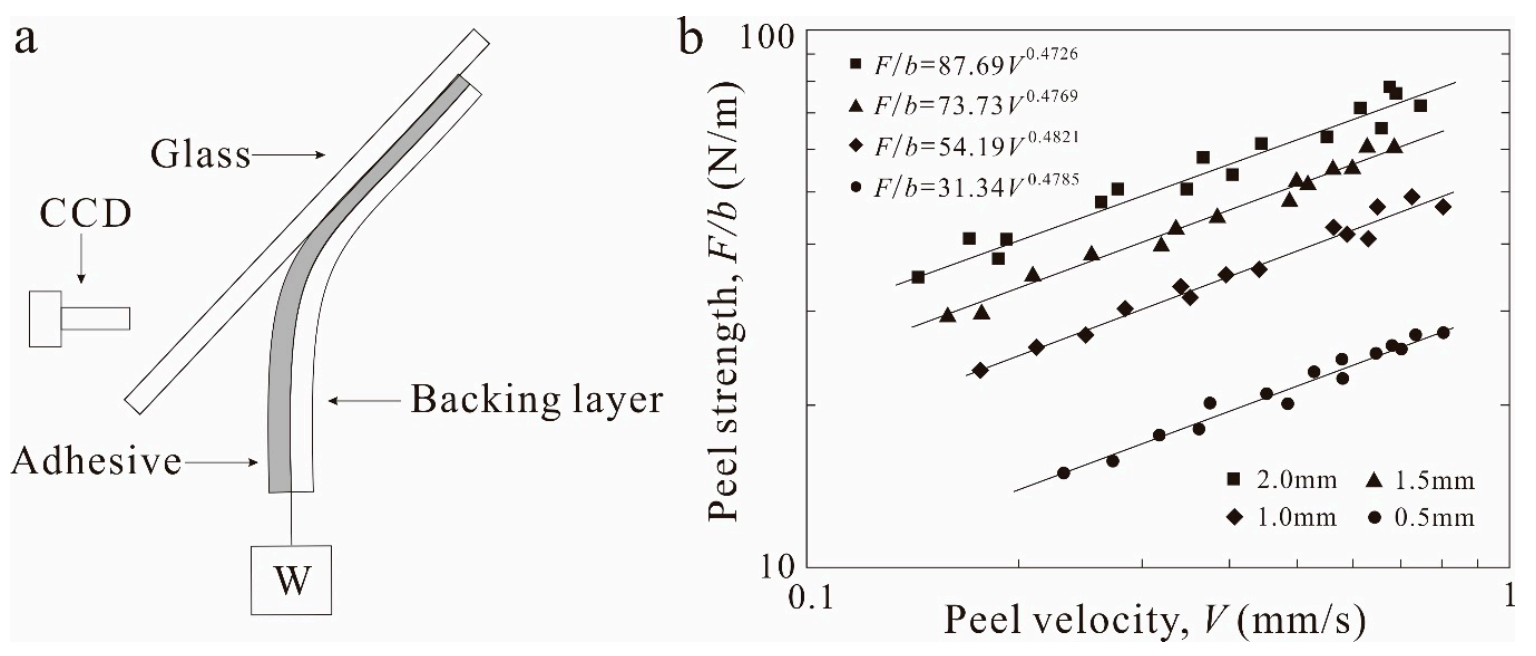

Figure 4. Results of the peeling test. (a) Schematic diagram of the peeling experiment; (b) The relationship between peel strength and peel velocity at a peeling angle of $30^{\circ}$ with different thicknesses of backing layer in log-log coordinates.

A linear relationship is found between the peel strength and the peel velocity in the log-log plot. Peel strength keeps increasing while thickness of the backing layer increases. The slopes of the four curves are almost the same, indicating that the physical properties of the adhesive are identical, while the differences between the intercepts indicate the different stiffness of the backing layer. The results agree with the experimental results obtained by Blum et al. [31] and Zhou et al. [33]. When the thickness of the backing layer is varied, the stiffness of the backing layer is changed, resulting in the increasing of the peel strength as stiffness increasing up until a saturation point (Figure 5). For the robot's footpad, the backing layer is indispensable, because of the stronger peeling strength. Thin backing layer effects not only in low peeling strength, which might be advantageous for contact breakage, but also in strong toe deformation during contact formation, which might prevent proper contact formation. 


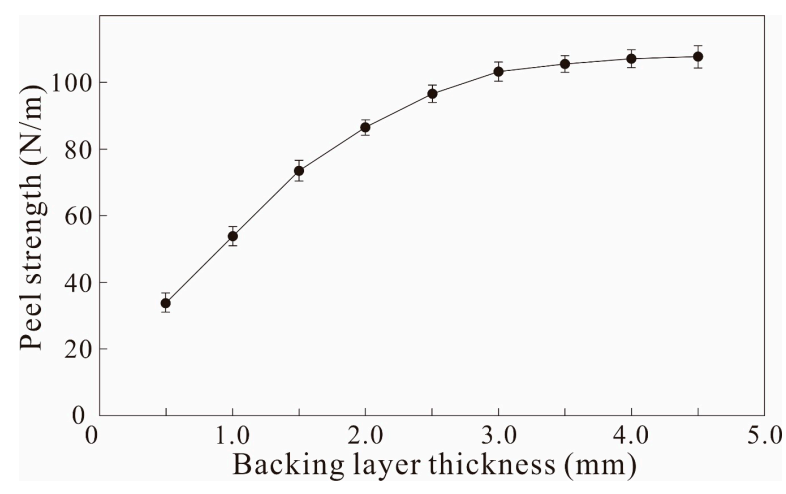

Figure 5. The influences of backing layer thickness on the peel strength at a peeling angle of $30^{\circ}$ and at a peeling velocity of $2 \mathrm{~mm} / \mathrm{s}$.

\section{Experiments on Robots}

\subsection{Climbing Ability with Different Toes}

Helium balloons which are fixed on the center of mass of the robot were used to reduce gravity. The climbing abilities of the robot under different conditions under reduced gravity were tested to verify the performance of the toes (Figure 6a). Here we define a ratio $(\mu)$ of reduced gravity (RRG):

$$
\mu=\frac{G-G_{r}}{G}
$$

Here $G$ is the weight of the robot, $G_{r}$ is the weight of the robot under reduced gravity conditions. All experiments were conducted on a glass surface, the size of each toe is $2 \mathrm{~cm} \times 3 \mathrm{~cm}$.

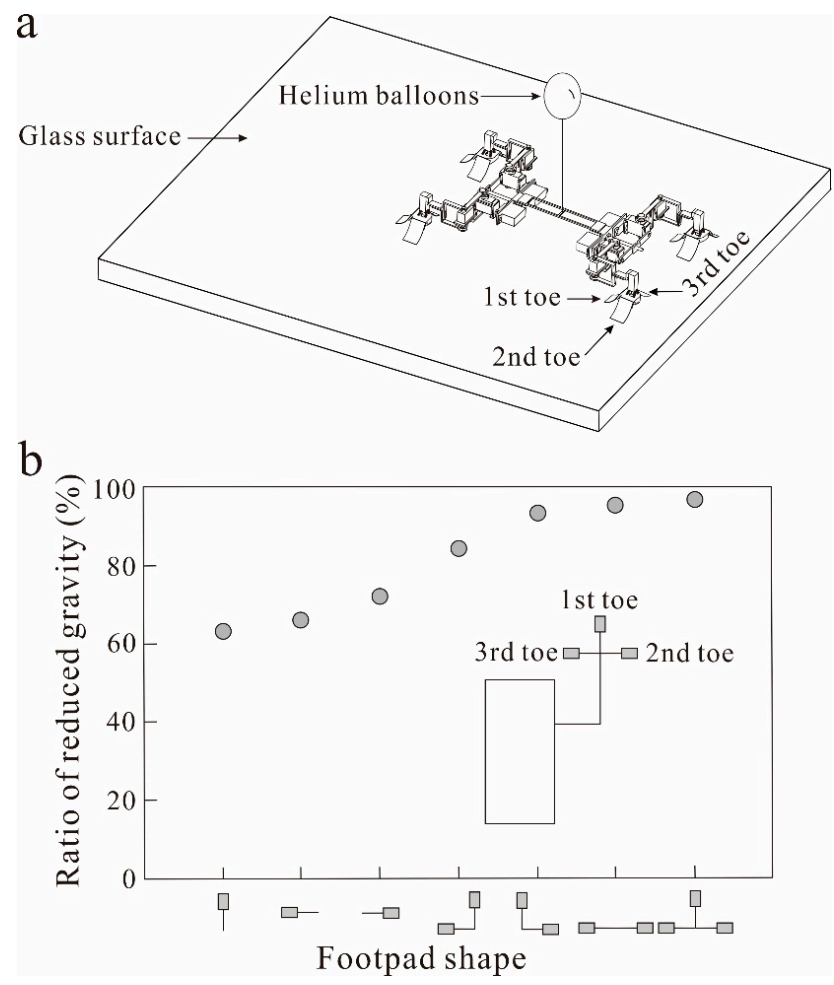

Figure 6. (a) Schematic of the robot climbing under reduced gravity; (b) Climbing ability with different footpad shape at different RRG. 
The results show the synergistic effect of different toes in one leg (Figure 6b). The 2nd and 3rd toes are mainly responsible for the improvement of the lateral balance of the robot by pulling inward toward the center of the leg. As the RRG increases, the role of lateral toes becomes even more important, because the robot needs to make transition from a crouched posture to a sprawled one, to ensure smaller peeling angle and prevent failure. This splaying behaviour is also observed in geckos [24] and tree frogs [34] when they face a stepper surface. The legs should generate large inward forces, in order to ensure that the angle of the pulling force remains small. Thus, the outward oriented toe, which can generate reliable shear force, is essential under a challenging condition, such as wall, ceiling and zero-g environment.

Figure 7 shows the robot climbing at a RRG of $94 \%$ by using the 1st and 2 nd toes at each foot (Supplementary video 1). Even without the 3rd toe, the robot can climb stably. The robot can climb under a lower ratio of the reduced gravity, when the 3rd toe is added, because an extra toe leads to the peeling force increase, in turn leading to an increased peeling time that ensure stability of locomotion. The ratio of reduced weight increases only slightly with an addition of the 3rd toe (Figure 6b).

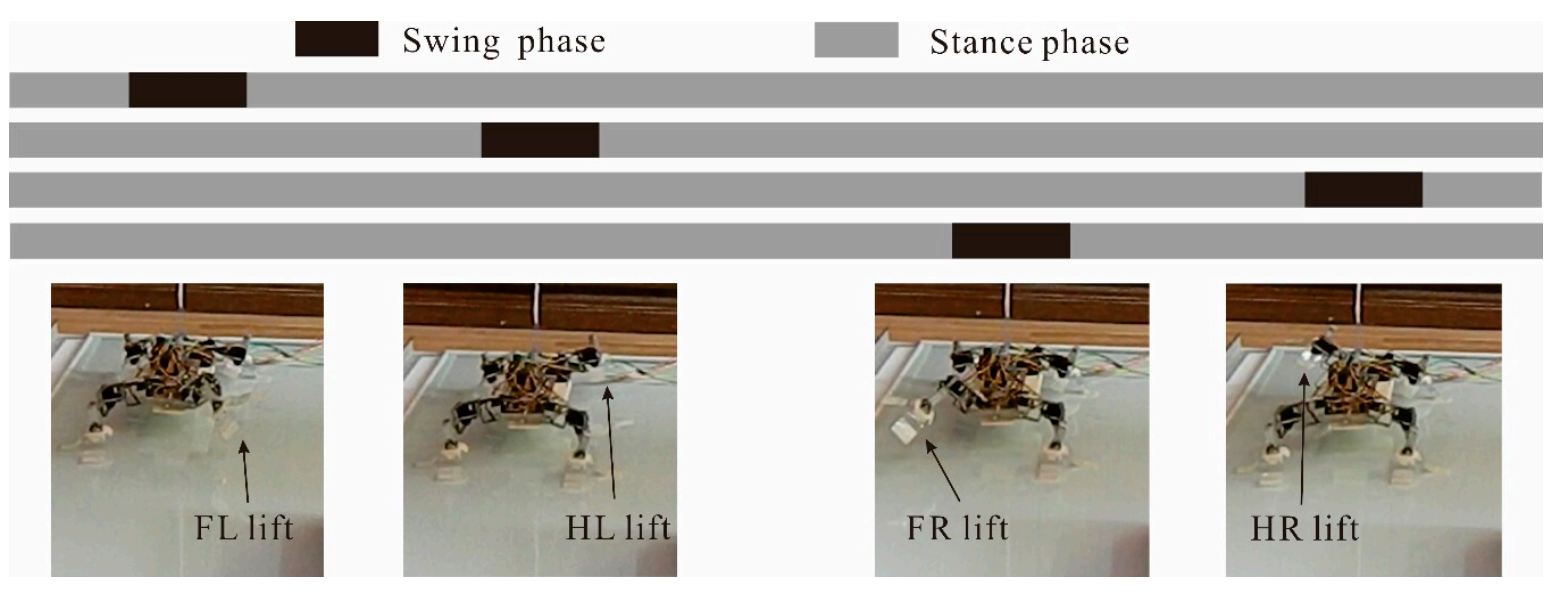

Figure 7. Robot climbing on a glass surface at a RRG of $94 \%$. Photos show different legs in swing phase. FL: Front left, HL: Hind left, FR: Front right, HR: Hind right.

\subsection{Climbing Performance with Lateral Impact}

We push the balloons in side direction slowly and quickly, respectively, to test the climbing performance at the lateral impact. When we pushed the balloons slowly, there was no slip observed during climbing. The climbing performance was not affected by such a lateral impact, and the robot could continue to move forward (Figure 8a) (Supplementary video 2). When we pushed balloons quickly, the robot slipped off, but did not fall down within a short distance in lateral direction (Figure 8b). Then, the robot recovered quickly and continued forward motion. These results demonstrate an enhanced self-balancing ability of the robot with multiple toes. It demonstrated feasibility of the multi-toe footpad and its higher reliability in the climbing performance under reduced gravity.

Kendall's peeling model demonstrates that peel strength increases with increasing Young's modulus of the backing layer. When the PVS is peeled off from a substrate, fibrills are stretched and deformed until the tension stress surpasses the pull-off stress. Under this condition, detachment is started. For the fibrillar surface, the stretching velocity of the fibril influences the resulting peel force [29]. Previous studies have shown that the relationship between the peel force and the peel velocity at the same peel angle can be described by [30-32]. 


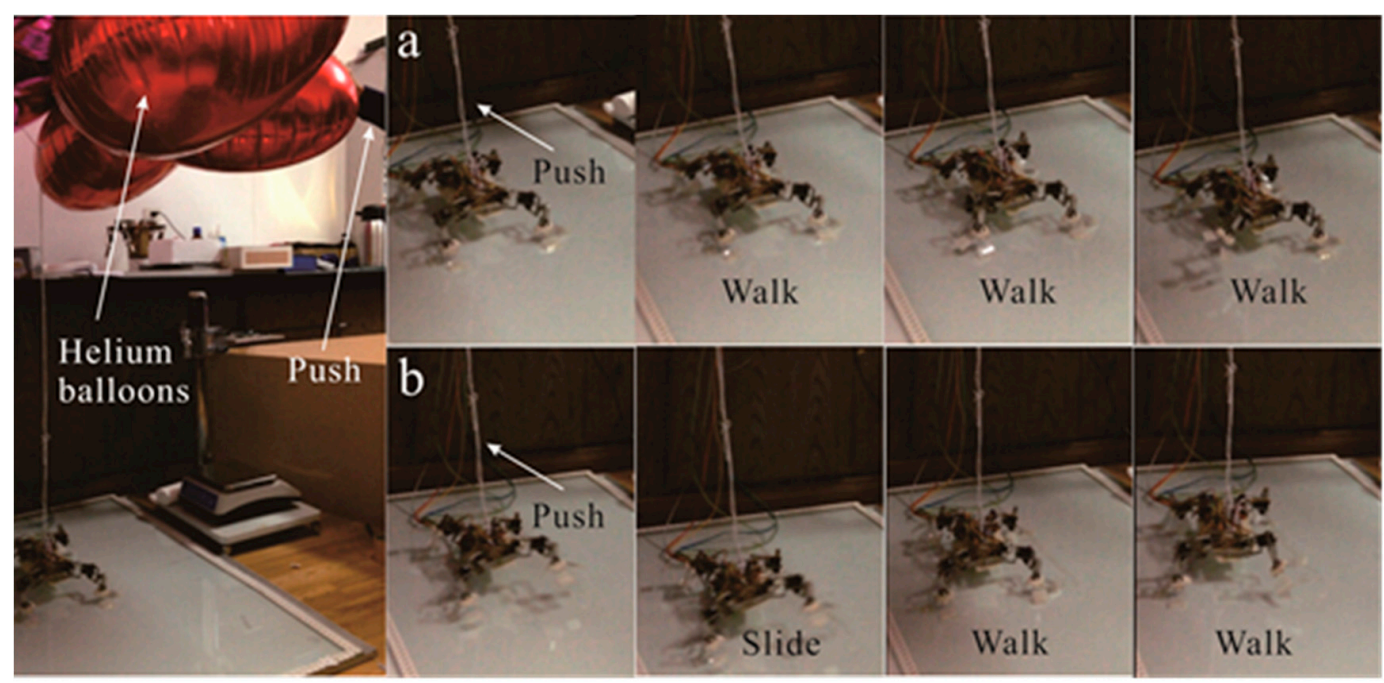

Figure 8. Robot climbing on glass surface with lateral impact at a RRG of $94 \%$.

\subsection{Climbing Ability of the Robot}

In order to test climbing ability of the robot under reduced gravity, the balloons were used to fully balance the robot's weight, as shown in Figure 9. The size of the toe is $2 \mathrm{~cm} \times 4 \mathrm{~cm}$. Our experiment shows that the robot can climb under mimic zero gravity at the velocity of up to $1 \mathrm{~cm} / \mathrm{s}$ with diagonal gait (Duty factor is 0.6 ). When the velocity of the increased up to $2 \mathrm{~cm} / \mathrm{s}$, the robot was slipped and become unstable. This is because as the velocity increases, peel strength also increases, which leads to larger peeling force.

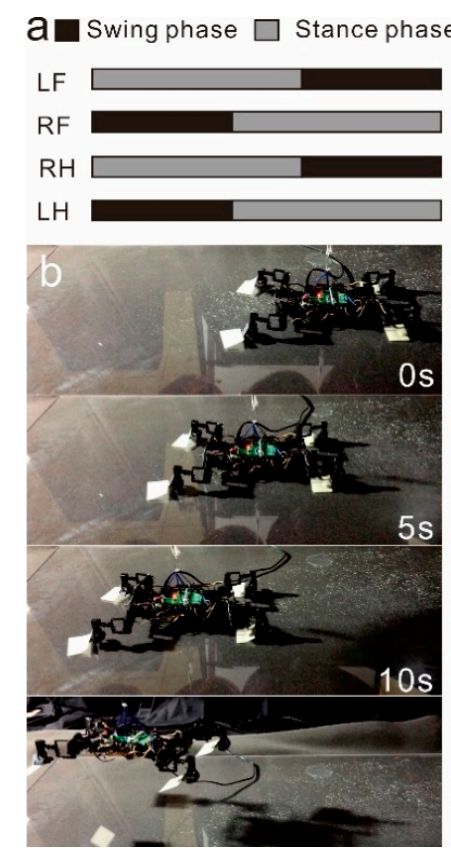

Figure 9. Experiments of robot under micro-gravity environment with diagonal gait.

\section{Conclusions and Future Work}

This paper presents the biologically inspired design of the footpad for a legged climbing robot. The biomimetic MSAMS-based tape was used here to design a bio-inspired footpad with multiple toes. The experimental tests demonstrated that the robot can climb undermimic zero gravity at the 
velocity of up to $1 \mathrm{~cm} / \mathrm{s}$, and even resist lateral impact. The major contribution of this work is the application of the biological principle of multi-toe footpad to the legged robot, and the demonstration of the feasibility and reliability of this design. To the best of our knowledge, the legged robot, climbing under nearly zero gravity, was demonstrated here for the first time. We also explored the effect of the (1) preload, (2) preload velocity, (3) peeling velocity, (4) thickness of the backing layer, and (5) collective effect of multiple toes in one leg on the reliability of the robot locomotion.

Further optimization of the robot is planned to improve its performance in climbing on vertical and inverted surfaces. Our preliminary studies have shown that it also can climb on incline surface of $75^{\circ}$ without tail, but failed to climb the inverted surface due to its heavy weight. The decrease of the robot mass will aid in an increase of the climbing ability to more challenged surfaces. In addition, the climbing speed is planned to be improved by using dynamical trotting gait.

Supplementary Materials: The following are available online at http:/ /www.mdpi.com/2076-3417/8/1/114/s1.

Acknowledgments: This work is supported by the National Natural Science Foundation of China (No. 51435008 and 31601870), the Doctoral Fund of Ministry of Education of China (No. 20123218110031), the Natural Science Foundation of Jiangsu Province, China (No. SBK2016040649) and the National Defense Pre-Research Foundation of China (No. B2520110013). This work is also partially supported by Jiangsu Province Friendship Award to S N Gorb.

Author Contributions: Zhongyuan Wang and Zhendong Dai conceived and designed the experiments; Zhongyuan Wang performed adhesion performances test, Zhongyuan Wang and Stanislav N. Gorb performed robot experiment, Zhongyuan Wang analyzed the data; Zhongyuan Wang, Zhouyi Wang, Zhendong Dai and Stanislav N. Gorb wrote the paper.

Conflicts of Interest: The authors declare no conflict of interest. The founding sponsors had no role in the design of the study; in the collection, analyses, or interpretation of data; in the writing of the manuscript, and in the decision to publish the results.

\section{References}

1. Arzt, E.; Gorb, S.; Spolenak, R. From micro to nano contacts in biological attachment devices. Proc. Natl. Acad. Sci. USA 2003, 100, 10603-10606. [CrossRef] [PubMed]

2. Cutkosky, M.R.; Kim, S. Design and fabrication of multi-material structures for bioinspired robots. Philos. Trans. R. Soc. A 2009, 367, 1799-1813. [CrossRef] [PubMed]

3. Gorb, S.N.; Sinha, M.; Peressadko, A.; Daltorio, K.A.; Quinn, R.D. Insects did it first: A micropatterned adhesive tape for robotic applications. Bioinspir. Biomim. 2007, 2, S117. [CrossRef] [PubMed]

4. Sitti, M.; Fearing, R.S. Synthetic gecko foot-hair micro/nano-structures for future wall-climbing robots. In Proceedings of the IEEE International Conference on Robotics and Automation (ICRA '03), Taipei, Taiwan, 14-19 September 2003.

5. Campo, D.A.; Greiner, C.; Alvarez, I.; Arzt, E. Patterned surfaces with pillars with controlled 3D tip geometry mimicking bio-attachment devices. Adv. Mater. 2007, 19, 1973-1977. [CrossRef]

6. Lee, D.Y.; Lee, D.H.; Lee, S.G.; Cho, K. Hierarchical geckoinspired nanohairs with a high aspect ratio induced by nanoyielding. Soft Matter 2007, 8, 4905-4910. [CrossRef]

7. Davies, J.; Haq, S.; Hawke, T.; Sargent, J.P. A practical approach to the development of a synthetic gecko tape. Int. J. Adhes. Adhes. 2009, 29, 380-390. [CrossRef]

8. Haefliger, D.; Boisen, A. Three-dimensional microfabrication in negative resist using printed masks. J. Micromech. Microeng. 2006, 16, 951-957. [CrossRef]

9. Sameoto, D.; Menon, C. Direct molding of dry adhesives with anisotropic peel strength using an offset lift-off photoresist mold. J. Micromech. Microeng. 2009, 19, 115026. [CrossRef]

10. Gorb, S.; Varenberg, M.; Peressadko, A.; Tuma, J. Biomimetic mushroom-shaped fibrillar adhesive microstructure. J. R. Soc. Interface 2007, 4, 271-275. [CrossRef] [PubMed]

11. Kim, S.; Sitti, M. Biologically inspired polymer microfibers with spatulate tips as repeatable fibrillar adhesives. Appl. Phys. Lett. 2006, 89, 261911. [CrossRef]

12. Daltorio, K.A.; Wei, T.E.; Horchler, A.D.; Southard, L.; Wile, G.D.; Quinn, R.D.; Gorb, S.N.; Ritzmann, R.E. Mini-whegs TM climbs steep surfaces using insect-inspired attachment mechanisms. Int. J. Robot. Res. 2009, 28, 285-302. [CrossRef] 
13. Murphy, M.P.; Kute, C.; Mengüç, Y.; Sitti, M. Waalbot II: Adhesion recovery and improved performance of a climbing robot using fibrillar adhesives. Int. J. Robot. Res. 2011, 30, 118-133. [CrossRef]

14. Unver, O.; Uneri, A.; Aydemir, A.; Sitti, M. Geckobot: A gecko inspired climbing robot using elastomer adhesives. In Proceedings of the 2006 IEEE International Conference on Robotics and Automation (ICRA '06), Orlando, FL, USA, 15-19 May 2006.

15. Li, Y.; Ahmed, A.; Sameoto, D.; Menon, C. Abigaille II: Toward the development of a spider-inspired climbing robot. Robotica 2012, 30, 79-89. [CrossRef]

16. Henrey, M.; Ahmed, A.; Boscariol, P.; Shannon, L.; Menon, C. Abigaille-III: A versatile, bioinspired hexapod for scaling smooth vertical surfaces. J. Bionic Eng. 2014, 11, 1-17. [CrossRef]

17. Kim, S.; Spenko, M.; Trujillo, S.; Heyneman, B.; Santos, D.; Cutkosky, M.R. Smooth vertical surface climbing with directional adhesion. IEEE Trans. Robot. 2008, 24, 65-74.

18. Dai, Z.; Sun, J. A biomimetic study of discontinuous-constraint metamorphic mechanism for gecko-like robot. J. Bionic Eng. 2007, 4, 91-95. [CrossRef]

19. Wang, Z.; Dai, Z.; Yu, Z.; Shen, D. Optimal attaching and detaching trajectory for bio-inspired climbing robot using dry adhesive. In Proceedings of the 2014 IEEE/ASME International Conference on Advanced Intelligent Mechatronics (AIM 2014), Besançon, France, 8-11 July 2014.

20. Autumn, K.; Niewiarowski, P.H.; Puthoff, J.B. Gecko adhesion as a model system for integrative biology, interdisciplinary science, and bioinspired engineering. Annu. Rev. Evol. Syst. 2014, 45, 445-470. [CrossRef]

21. Wang, Z.; Dai, Z.; Ji, A.; Ren, L.; Xing, Q.; Dai, L. Biomechanics of gecko locomotion: The patterns of reaction forces on inverted, vertical and horizontal substrates. Bioinspir. Biomim. 2015, 10, 016019. [CrossRef] [PubMed]

22. Tian, Y.; Pesika, N.; Zeng, H.; Rosenberg, K.; Zhao, B.; McGuiggan, P.; Israelachvili, J. Adhesion and friction in gecko toe attachment and detachment. Proc. Natl. Acad. Sci. USA 2006, 103, 19320-19325. [CrossRef] [PubMed]

23. Autumn, K.; Dittmore, A.; Santos, D.; Spenko, M.; Cutkosky, M. Frictional adhesion: A new angle on gecko attachment. J. Exp. Biol. 2006, 209, 3569-3579. [CrossRef] [PubMed]

24. Autumn, K.; Hsieh, S.T.; Dudek, D.M.; Chen, J.; Chitaphan, C.; Full, R.J. Dynamics of geckos running vertically. J. Exp. Biol. 2006, 209, 260-272. [CrossRef] [PubMed]

25. Chen, J.J.; Peattie, A.M.; Autumn, K.; Full, R.J. Differential leg function in a sprawled-posture quadrupedal trotter. J. Exp. Biol. 2006, 209, 249-259. [CrossRef] [PubMed]

26. Lepore, E.; Pugno, F.; Pugno, N.M. Optimal angles for maximal adhesion in living tokay geckos. J. Adhes. 2012, 88, 820-830. [CrossRef]

27. Goldman, D.I.; Chen, T.S.; Dudek, D.M.; Full, R.J. Dynamics of rapid vertical climbing in cockroaches reveals a template. J. Exp. Biol. 2006, 209, 2990-3000. [CrossRef] [PubMed]

28. Kendall, K. Thin-film peeling: The elastic term. J. Phy. D Appl. Phys. 1975, 8, 1449-1452. [CrossRef]

29. Zhou, M.; Pesika, N.; Zeng, H.; Wan, J.; Zhang, X.; Meng, Y.; Wen, S.; Tian, Y. Design of gecko-inspired fibrillar surfaces with strong attachment and easy-removal properties: A numerical analysis of peel-zone. J. R. Soc. Interface 2012, 9, 2424-2436. [CrossRef] [PubMed]

30. Amouroux, N.; Petit, J.; Léger, L. Role of interfacial resistance to shear stress on adhesive peel strength. Langmuir 2001, 17, 6510-6517. [CrossRef]

31. Blum, F.D.; Metin, B.; Vohra, R.; Sitton, O.C. Surface segmental mobility and adhesion-Effects of filler and molecular mass. J. Adhes. 2006, 82, 903-917. [CrossRef]

32. Marin, G.; Derail, C. Rheology and adherence of pressure-sensitive adhesives. J. Adhes. 2006, 82, 469-485. [CrossRef]

33. Zhou, M.; Tian, Y.; Pesika, N.; Zeng, H.; Wan, J.; Meng, Y.; Wen, S. The extended peel zone model: Effect of peeling velocity. J. Adhes. 2011, 87, 1045-1058. [CrossRef]

34. Endlein, T.; Ji, A.; Samuel, D.; Yao, N.; Wang, Z.; Barnes, W.J.P.; Federle, W.; Kappl, M.; Dai, Z. Sticking like sticky tape: Tree frogs use friction forces to enhance attachment on overhanging surfaces. J. R. Soc. Interface 2013, 10, 20120838. [CrossRef] [PubMed]

(C) 2018 by the authors. Licensee MDPI, Basel, Switzerland. This article is an open access article distributed under the terms and conditions of the Creative Commons Attribution (CC BY) license (http://creativecommons.org/licenses/by/4.0/). 